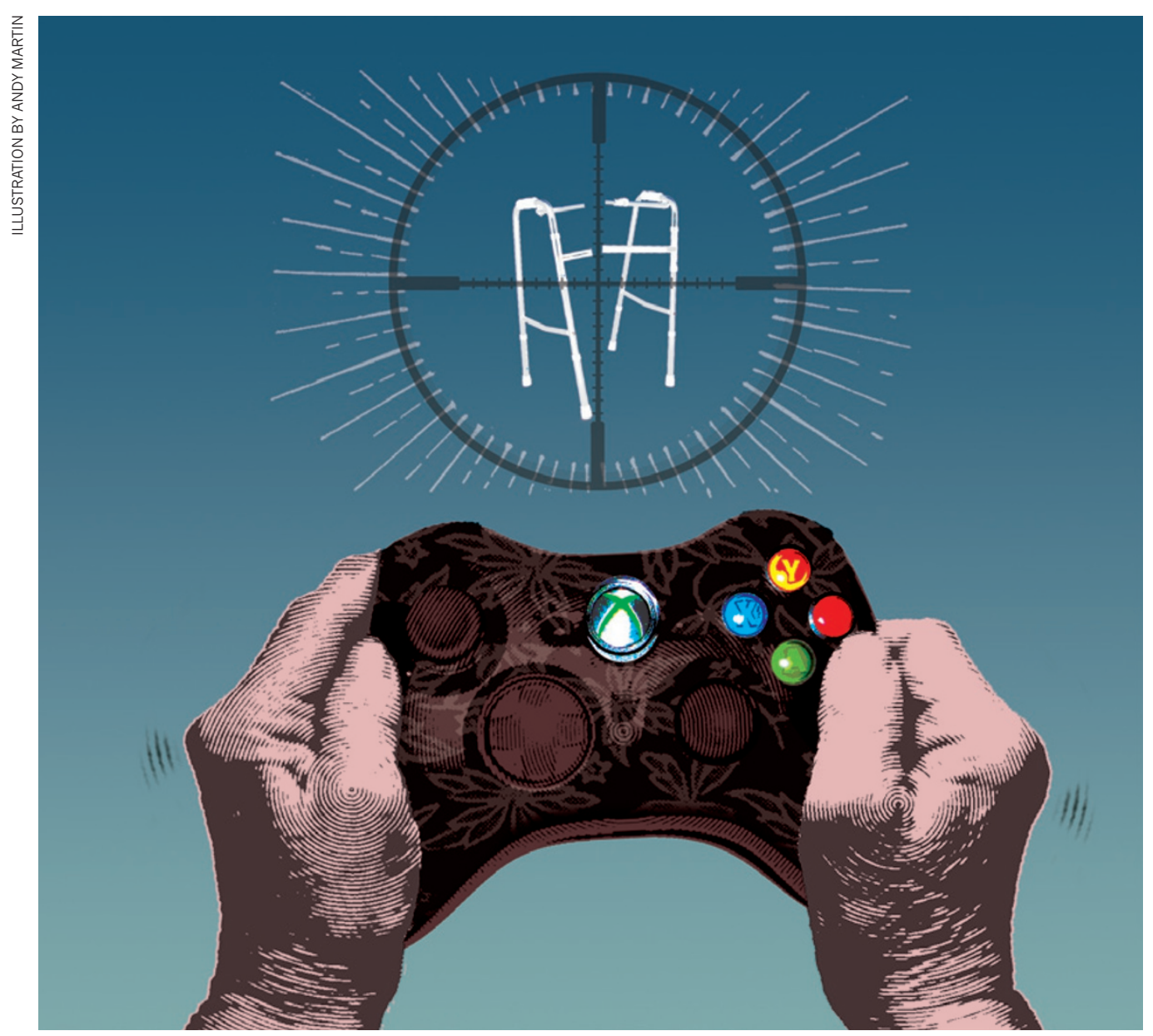

\title{
Games to do you good
}

\section{Neuroscientists should help to develop compelling video games that boost brain function and improve well-being, say Daphne Bavelier and Richard J. Davidson.}

$\mathrm{I}$ $\mathrm{n}$ the month after its release, the video game Call of Duty: Black Ops had been played for the equivalent of 68,000 years worldwide. Children in the United States played an average of 1 hour and 13 minutes of video games every day in 2009 - a nearly threefold increase from 10 years earlier ${ }^{1}$. And people of all ages are playing. Only $18 \%$ of US gameplayers are under 18 years old; nearly one-third are over 50 .

Video games are associated with a variety of negative outcomes, such as obesity, aggressiveness, antisocial behaviour and, in extreme cases, addiction ${ }^{2}$. At the same time, evidence is mounting that playing games can have beneficial effects on the brain.

After spending an hour a day, 5 days a week for 8-10 weeks spotting snipers and evading opponents in shooter games such as Call of Duty or Unreal Tournament, young adults saw more small visual details in the middle of clutter and more accurately distinguished between various shades of grey $^{3}$. After 10 hours stretched over 2 weeks spent chasing bad guys in mazes and labyrinths, players were better able to rotate an image mentally ${ }^{4}$, an improvement that was still present six months later and could be useful for activities as varied as navigation, research chemistry and architectural design. After guiding small rodents to a safe exit amid obstacles during a version of the game Lemmings that was designed to encourage positive behaviour, players were more likely in simulated scenarios to help another person after a mishap or to intervene when someone was being harassed ${ }^{5}$.

Because gaming is clearly here to stay, some scientists are asking how to channel people's love of screen time towards positive effects on the brain and behaviour by designing video games specifically intended to train particular aspects of behaviour and brain function. One game, for example, aims to treat depression by introducing cognitive behavioural therapy while users fight off negative thoughts in a fantasy world ${ }^{6}$. In Re-mission, young cancer patients blast cancer cells and fight infections and the side effects of therapy - all to encourage them to stick with treatment (see www.re-mission.net).

Last year, we and other scientists studying the brain met entertainment-media experts to discuss ways of using interactive technology such as video games to further our understanding of brain functions and to provide new, engaging rehabilitation tools, in particular for boosting attention and well-being. The meeting was hosted by the White House Office of Science and Technology Policy and sponsored by the US National Science Foundation (see go.nature. com/t9mvqc). 


\section{BRAIN GAME}

When searching for a particular object in a sea of shapes, people who played video games regularly showed less activation of the brain regions linked to attention, a sign that their brains were performing the task more efficiently.
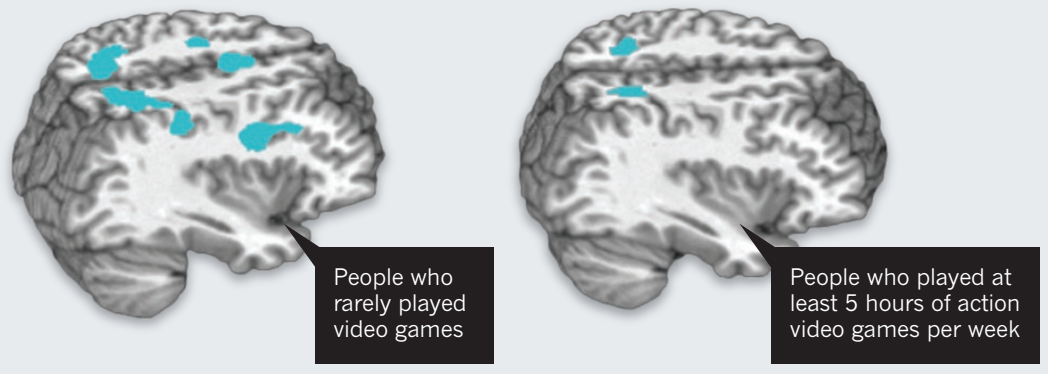

To make the sort of video games that might one day improve empathy and positive social interaction, or decrease anxiety and sharpen attention, the developments outlined below will be necessary.

\section{EXPLORING THE BRAIN}

First, game designers and brain scientists must determine which game components foster brain plasticity, to guide the design of tomorrow's video games. This is not intuitive. For instance, playing hours of action video games seems to have no effect on players' ability to react reflexively to loud noises, even though that type of attention is frequently solicited during the game ${ }^{3}$. By contrast, action gameplayers are better able to deliberately allocate their attention to one part of their environment, ignoring other sources of distraction (see 'Brain game'). This is a quality known as attentional control, and is not obviously associated with chasing zombies ${ }^{3}$.

Another key challenge is to map the effects of video games in the brain, because many overlapping skills are called on during even a few seconds of gaming. Neuroscience is still in its infancy when it comes to mapping complex behaviour, but video-game designers could create controls that help researchers to systematically vary how much of each skill is needed during gameplay, enabling them to map the corresponding brain circuit.

\section{WORKING TOGETHER}

Second, the next generation of neuroscientists must collaborate better with the games industry. Universities could facilitate links by creating new multidisciplinary programmes that subvert rigid departmental boundaries and bring together experts ranging from artists and psychologists to programmers and neuroscientists. Stronger links to professional game designers would also facilitate the development of therapeutic games that people actually want to play as much as Call of Duty.

The games industry could help tremendously by adding brain scientists to their teams. These researchers could assist the industry in enhancing players' experience, while gaining insights into players' behaviour by studying the industry's large gameplay records, which track players' actions and responses to various game components. Every year, Valve, a game company based in Bellevue, Washington, involves a psychology graduate student from the nearby University of Washington in Seattle in their gameplay research.

\section{GETTING TO MARKET}

Third, experts must develop a path for academics to bring potentially therapeutic games to market, similar to the translational process that takes drugs from the lab to the clinic. No such path exists for games, but some academics are trying. For example, neuroscientist Adam

Gazzaley and his colleagues at the University of California, San Francisco, have developed a game to ameliorate age-related decline in attention, by asking adults to play a

\section{"Many overlapping skills are called on during even a few seconds of gaming."} driving game in which they avoid various distractions along the way. Before and after playing, the researchers measure a player's brain function and test their ability to resist distraction.

Gazzaley is a founding adviser to a new company - Akili Interactive Labs in Boston, Massachusetts, for which one of us (D.B.) serves on the advisory board - that aims to pursue the development, large-scale validation and commercialization of this game to improve resistance to distraction.

\section{EXPERIMENTAL DESIGN}

Finally, those in the field should recognize that researching the impact of gaming on the brain is inherently difficult. Presenting a video or computer game to a participant in a research study necessarily requires that they are aware of the intervention. We must therefore develop standards to evaluate the impact of gameplay on brain and behaviour to avoid false claims of benefits. Control interventions should be matched to the experimental version on as many variables as possible, including the amount of gameplaying, the difficulty level and the interest value. Placebo controls are not possible, so optimal designs probably involve having several comparison groups, including an active gameplaying comparison and perhaps other, more typical interventions, such as drug therapy. And even if participants are not blinded, experimenters should be.

It is important to note that even if experts can design a new breed of video games that benefit brain function, it will not provide a carte blanche for video-game bingeing. Exposures that show beneficial effects -5 hours of gaming per week for 2-10 weeks - are a fraction of the time that most young gamers play. And violent, negative content can have detrimental effects on social and emotional skills, calling for much caution ${ }^{7}$. An important challenge for both academics and the games industry is to collaborate on the development of games as compelling as those in which many young people now indulge, but that help to cultivate positive qualities such as empathy and cooperation ${ }^{8,9}$.

Gradually, this work will begin to address the burning social question of how technology is having an impact on our brains and our lives, and enable us to make evidencebased choices about the technologies of the future, to produce a new set of tools to cultivate positive habits of mind.

Daphne Bavelier is in the Department of Brain and Cognitive Sciences, University of Rochester, Rochester, New York 14627-0268, USA, and the Department of Psychology and Education Sciences, University of Geneva, Switzerland. Richard J. Davidson is at the Center for Investigating Healthy Minds, University of Wisconsin, Madison, Wisconsin 53705-2280, USA. e-mails:daphne@bcs.rochester.edu; rjdavids@wisc.edu

1. Rideout, V. J., Foehr, U. G. \& Roberts, D. F. Generation M²: Media in the Lives of 8- to 18-YearOlds (Kaiser Family Foundation, 2010); available at go.nature.com/sb7pjp.

2. Strasburger, V. C., Jordan, A. B. \& Donnerstein, E. Pediatrics 125, 756-767 (2010).

3. Green, C. S. \& Bavelier, D. Curr. Biol. 22, R197R206 (2012).

4. Feng, J., Spence, I. \& Pratt, J. Psychol. Sci. 18, 850-855 (2007)

5. Greitemeyer, T. \& Osswald, S. J. Pers. Soc. Psychol. 98, 211-221 (2010).

6. Merry, S. N. et al. Br. Med. J. 344, e2598 (2012).

7. Bavelier, D., Green, C. S., Han, D. H., Renshaw, P. F. \& Merzenich, M. M. Nature Neurosci. 12, 763-768 (2011).

8. Davidson, R. J. \& McEwen, B. S. Nature Neurosci. 15, 689-695 (2012).

9. Klimecki, O. M., Leiberg, S., Lamm, C. \& Singer, T. Cerebral Cortex http://dx.doi.org/10.1093/ cercor/bhs142 (2012).

D. B. declares competing financial interests: see go.nature.com/jmunrh for details. 